\title{
Technologies de l'information et travail collectif médiatisé. Repenser la relation interpersonnelle
}

Isabelle Comtet

\section{(2) OpenEdition}

12 Journals

Édition électronique

URL : http://journals.openedition.org/communicationorganisation/2920

DOI : 10.4000/communicationorganisation.2920

ISSN : $1775-3546$

Éditeur

Presses universitaires de Bordeaux

Édition imprimée

Date de publication : 1 mai 2004

ISSN : 1168-5549

Référence électronique

Isabelle Comtet, «Technologies de l'information et travail collectif médiatisé. Repenser la relation interpersonnelle », Communication et organisation [En ligne], 24 | 2004, mis en ligne le 27 mars 2012 consulté le 19 avril 2019. URL : http://journals.openedition.org/communicationorganisation/2920 ; DOI : 10.4000/communicationorganisation.2920

Ce document a été généré automatiquement le 19 avril 2019

(c) Presses universitaires de Bordeaux 


\title{
Technologies de l'information et travail collectif médiatisé. Repenser la relation interpersonnelle
}

\author{
Isabelle Comtet
}

\section{Introduction}

1 Dans le contexte actuel qui est celui de la mondialisation des échanges économiques, culturels et sociaux, l'usage des technologies participe d'une activité sociale étendue, réticulaire et multiple. Les technologies dites "d'information et de communication" semblent favoriser, autoriser ou faciliter les liens interpersonnels dans un contexte où les distances spatiales et temporelles sont de plus en plus grandes, finalement, ces TIC réorganisent d'une certaine façon les espaces sociaux autour de l'immédiateté (ou de la quasi-immédiateté) de la multiplicité et d'une prétendue facilité de communication.

2 Un de ces espaces sociaux semble tout particulièrement lié à ces enjeux : c'est le domaine de l'activité professionnelle. Au regard des enjeux évoqués, ce domaine pourrait développer de nouvelles formes d'organisation du travail propices à la mise en œuvre de liens interpersonnels médiatisés. C'est ce que la littérature scientifique anglaise, en tant que précurseur, a appelé le "Computer Supported Collaborative Word» ou Travail collaboratif assisté par ordinateur. Les chercheurs de ce domaine travaillent sur des outils spécifiques comme les logiciels de Travail Collaboratif qui sont destinés à faciliter plus précisément le travail d'équipe, t'es formes de travail assistées par ordinateur constituent des systèmes d'informations destinés au travail « électronique ». Ce qui est ici en jeu, c'est l'émergence d'un éventuel changement de mode de travail et de construction de la relation interpersonnelle en lien avec l'usage de nouvelles technologiques. Cet usage est alors considéré comme construit lié :

- au statut de la technique (donc aux enjeux de celle-ci), 
- au statut des objets (en considérant par exemple la communication comme une valeur positive et instrumentale).

- et au statut du quotidien (qui prend en compte les TIC par rapport aux pratiques existantes). celui des télé-activités de manière globale. Mais, plus précisément, nous prenons en compte les activités distantes mettant en jeu plusieurs acteurs devant agir ensemble pour réaliser une activité professionnelle qui se veut collective, et pour laquelle les contraintes d'espaces et de temps impliquent l'utilisation d'un système collaboratif.

\section{Problématisation et méthodologie}

5 Il s'agit ainsi pour nous, dans un premier temps, d'analyser les usages que font des acteurs interdépendants de certains systèmes collaboratifs. Ensuite, une meilleure compréhension de ces usages nous permet de porter la réflexion sur une pratique technologique pensée comme un construit social. Ce construit est le fruit du lien entre le dispositif technique, les éléments organisationnels du contexte et les comportements des acteurs en situation.

6 Dès lors, partant du principe que la problématique des usages des systèmes collaboratifs implique une activité sociale au sein d'une organisation professionnelle, nous avons choisi de mener cette recherche à partir d'une double clé d'analyse relevant de la sociologie des organisations et de la sociologie pragmatique, La sociologie des organisations permet de réfléchir sur le fonctionnement de la vie interne d'un groupe, sur son système de relation. Nous avons pu prendre ainsi en considération le questionnement sur le contexte d'action collectif qui concerne le fonctionnement du groupe dans l'organisation. Cette approche théorique nous a apporté un ensemble d'explications quant au « fonctionnement » (c'est-à-dire au comportement, aux attitudes, aux réactions) d'un individu dans une organisation du travail.

7 Parallèlement, la sociologie pragmatique a focalisé aussi notre réflexion sur la place centrale du contexte pour réaliser une activité sociale à l'aide d'un dispositif sociotechnique, c'est-à-dire d'un système mêlant la dimension sociale et la dimension technologique dans la mesure où elles sont en interaction constante. Nous centrons ici notre questionnement sur le contexte d'action individuelle qui recouvre le rapport de l'acteur à l'outil. Cette approche théorique oriente notre travail vers le caractère interactionnel et collectif de ces situations de travail distant.

8 Une telle problématisation de l'objet d'étude nous amène à poser comme hypothèse centrale que l'usage d'un système collaboratif pour la réalisation d'un travail distant semble nécessiter la maîtrise complémentaire et parallèle du contexte d'action collectif et du contexte d'action individuel qui est celui du rapport de l'acteur à l'outil.

9

différents : 
10 Le premier mettait en jeu une expérimentation au cours de laquelle un groupe de chercheurs devait réaliser un document écrit à l'aide d'une conférence télématique. sur l'activité de groupe et la synergie des compétences émanant de celui-ci (Galegher. kraut. Egido. 1995) ${ }^{1}$. Le groupware suppose donc la formation d'une équipe ${ }^{2}$ à part entière. Celle-ci habituellement, est liée à l'existence de buts communs, à la volonté des acteurs de travailler ensemble, à la prise en charge de différents rôles et à l'émergence d'un intermédiaire entre les participants pour gérer et conduire le groupe (Crozier. Friedberg. 1977) ${ }^{3}$. On peut penser que l'usage de cet outil va donc probablement entraîner des modalités organisationnelles spécifiques, dans des structures plus habituées aux activités individuelles, même coordonnées entre elles.

\section{Caractéristiques organisationnelles}

recomposition d'un groupe d'acteurs professionnels se réalise virtuellement au travers d'une communication sociale médiatisée qui offre aux acteurs la possibilité de travailler au mieux, quelles que soit les distances spatiale et temporelle. En effet, on observe que le groupware. via l'ordinateur, serait générateur de liens sociaux : «Connaitre les capacités des personnes avec qui l'on travaille, aide. Le groupware facilite cela en améliorant les communications. Si le flot des informations [...] est fluide et efficace, alors les personnes contribuent ou travail en donnant le meilleur d'elles-mêmes. $»^{4}($ Opper. Fersko-Weiss. 1992) dans la mesure où la forme de communication proposée par l'outil (visuelle, auditive, orale ou seulement écrite) véhicule les marques du social et forme une situation analogue à celle du face-à-face en terme de choix de vocabulaire, de jeu de rôle...dont on sait les caractéristiques bénéfiques à une communication de bonne qualité (Goffman. 1973) 

compétences originales comme la connaissance de l'outil, le partage de l'information, la participation effective de tous les acteurs malgré la difficulté à travailler avec une technologie, et l'implication d'un participant nouveau avec un statut et un rôle spécifique: l'animateur de groupe médiatisé, ligure originale et essentielle sur l'importance de laquelle nous reviendrons, ce rôle est d'autant plus important que les systèmes collaboratifs impliquent avant tout une communication distante qu'il semble nécessaire d'apprendre à gérer (Périn. Gensollen. 1992) ${ }^{7}$ Les caractéristiques spécifiques à cet outil tendent, dans leur principe, à favoriser la synergie de compétences - autrement éclatées - et à rendre le groupe (et par là même, l'organisation à laquelle il est lié) plus efficace. Dans ces conditions seulement l'action aboutit à des résultats utiles.

au-delà de ces caractéristiques professionnelles, on peut s'interroger sur l'usage d'un tel outil technologique au niveau des modalités communicationnelles qui peuvent modifier ou transformer la façon de réaliser un travail à plusieurs.

\section{Modalités communicationnelles}

L'ensemble des caractéristiques liées à l'usage du groupware et au groupware lui-même, sont à mettre en parallèle avec les différentes modalités communicationnelles que propose ce type de travail. En effet, les canaux de communication ne sont pas de même nature selon les groupwares et ne généreraient pas a priori les mêmes liens sociaux, qu'il s'agisse d'une communication synchrone $^{8}$. quasi-synchrone ${ }^{9}$ ou asynchrone ${ }^{10}$.

21 Certains groupwares favorisent une communication synchrone, c'est-à-dire immédiate. Cette situation est liée à l'usage d'une multiplicité de canaux de communication. Ceux-ci pensent en effet être visuels ou audio-oraux. Ce type de dispositif configure alors une situation de travail de groupe proche du face-à-face. En terme de choix du canal de communication, ces groupwares permettent aux acteurs «d'auto-gérer » leur activité dans la mesure où ils peinent adapter leur travail aux modes de communication qui leur semblent pertinents ou familiers. Cependant, cette tonne de communication synchrone implique, dans le cas d'un travail collectif distant, que les acteurs choisissent un canal de communication qui convient à tous.

22 La gestion à l'intérieur du cadre structurel défini ne peut plus être seulement une activité individuelle. Elle doit devenir le fruit d'un acteur collectif et les acteurs doivent acquérir une réelle autonomie organisationnelle. Par ailleurs, la possibilité de choisir des canaux de communication qui "recréent» une situation de travail de groupe, facilite les discussions. Ces dernières sont propices aux confrontations et aux négociations. Ces espaces de communication des informations permettent en effet l'acquisition d'une certaine autonomie cognitive grâce à la mutualisation des connaissances.

Un autre type de communication est autorisé via l'utilisation de certains autres groupwares de type quasi-synchrone. La communication y est souvent comparée à celle en temps différé : elle permet d'échanger des informations très rapidement au cours d'une activité mais dans un temps qui n'est pas simultané. Elle implique nécessairement une communication pur l'écrit. Cette dernière, fondamentalement différente de celle caractéristique d'une situation de travail en face-à-face, semble supposer une organisation explicite de l'activité et une distribution précise des rôles et fonctions de chacun (Finholt. Sproull. Kiesler. 1995) ${ }^{11}$ pour l'efficacité du groupe. Ces auteurs expliquent à propos de ces systèmes collaboratifs : «[...] Il y a certainement une relation entre

Communication et organisation, 24 | 2012 
la capacité managériale et le volume de courrier électronique du groupe; Jonc, Je bons managers déterminent comment exploiter la technologie pour que le groupe en bénéficie directement $»^{12}$. (1995).

Actuellement une majorité des groupwares utilisés en milieu professionnel favorise une communication totalement asynchrone. Ils véhiculent, comme la précédente technologie évoquée, les informations par l'écrit, mais dans un temps différé. Ces groupwares asynchrones guident beaucoup plus l'activité que la communication quasi-synchrone. En effet, selon les observateurs, les acteurs seraient plus « contraints », que pour l'utilisation de systèmes multimodaux, de suivre des protocoles définis à l'avance par l'outil pour communiquer.

On constate ainsi que la communication par groupware semble, selon le dispositif en place, avoir un double effet : soit, elle facilite les relations interpersonnelles et l'efficacité dans la mesure où des canevas d'activités sont prévus pour organiser l'activité : soit, elle contraint trop les relations et dans ce cas, risque de ne générer qu'une communication pré-déterminée autoritairement, en sapant la capacité d'innovation dans l'activité du groupe.

\section{Comprendre l'interaction médiastée}

Nous avons parlé de règles de fonctionnement et de structuration des activités : du point de vue des différents partenaires sociaux : il est clair que globalement, les règles sont destinées à supprimer les sources d'incertitudes : Crozier et friedberg le soulignent : «Il est généralement admis que la règle est un moyen aux mains du supérieur pour obtenir un comportement conforme de la part de ses subordonnés. » (1977) ${ }^{13}$. Les règles fixent a priori la contribution des exécutants en définissant plus ou moins précisément des tâches ou des missions, et de fixer l'espace de travail dans lequel des acteurs sont affectés (De Terssac. 1992 ${ }^{14}$ Comment jouer ce processus de régulation dans le cadre d'un travail à visée collaborative?

\section{La régulation par les rôles}

Le choix d'un mode de travail collaboratif implique une distribution de rôles sociaux plus ou moins prescrits par l'organisation au sein du collectif.

Ils correspondent néanmoins, pour ceux qui en sont investis, à un ensemble d'obligations en fonction de leur position dans le système social. Le but du rôle est de lier un individu à la réalisation d'un projet collectif.

Ainsi, la manière dont est structurée une organisation, la mise en place de rôles et de règles, le type de communication choisie (très formalisée ou non) contribuent à «[...] la constitution d'un ensemble de normes et de valeurs, de représentations partagées, de façon de sentir, penser et agir " (Lafaye. 1996) ${ }^{15}$ L'institution produit ainsi des éléments de sociabilité, d'identités, de-valeurs, qui peuvent également, dans une certaine mesure «façonner » les acteurs ou du moins déterminer en partie la manière dont l'activité est réalisée. Il ne faut donc pas penser que l'organisation des rapports humains soit uniquement réductible à des conduites prescrites, rationnellement déterminées par l'entreprise. «Conditionnées, bien sûr. par le faisceau de contraintes de toutes sortes, caractéristiques d'une situation donnée, les actions et interactions [ces solutions] ne s'en déduisent pas mécaniquement Elles sont aussi 
l'expression des capacités relationnelles, c'est-à-dire culturelles, des individus" (Crozier. friedberg. 1977) ${ }^{16}$. Sans oublier que les comportements des acteurs relèvent aussi de leur histoire personnelle. Celle-ci filtre en quelque sorte leur perception des situations (Friedberg. 1993 $)^{17}$. C'est pourquoi une organisation ne peut déterminer à l'avance toutes les conditions de réalisation d'une activité collective, a fortiori lorsqu'elle a l'ambition de mettre en synergie un ensemble d'acteurs interdépendants professionnellement, collectifs mais également individualisés par leurs expériences.

\section{L'importance de l'appropriation et de l'autonomie}

l'intériorisation (ou non) des valeurs de l'organisation (par le biais de la culture d'entreprise par exemple). Cependant, la communication médiatisée au sein d'un collectif professionnel implique, pensons-nous, que l'acteur s'approprie l'espace de travail, le fait passer dans la sphère privée-dans le sens où Fisher souligne alors qu'il s'agit « [...] de faire sien, de s'attribuer la propriété de quelque chose, même si cela ne nous appartient ailleurs fortement «située » dans la mesure où les conduites de l'acteur renvoient à une socialisation historiquement construite, à la perception de ses opportunités et aux contraintes du contexte d'action. La rationalité est ici culturelle, contextuelle et contingente. "Les acteurs sont contingents car ils n'existent pas en dehors de leur contexte d'action dans lequel ils évoluent et dont la structuration conditionne la rationalité et leurs actions tout en étant façonnée en retour par elles" (Friedberg. 1993) ${ }^{19}$. On observe que l'idée qu'implique la rationalité limitée et située n'est pas sans rappeler la théorie de l'action située de Suchman dans laquelle les individus, la tâche, le mode de médiatisation s'influencent réciproquement. En effet, les individus sont placés dans des situations où lu plupart du temps rien ne leur appartient ni du point de vue matériel, ni du point de vue immatériel : ni la technologie, ni l'information qui circule au travers ne leur est propre. L'appropriation a donc ici une fonction nettement régulatoire. Elle est une intervention active de l'individu sur les lieux et technologies de travail dans la mesure où elle implique un ajustement entre l'acteur et l'organisation concernant la nature et les modalités de réalisation de l'activité. L'autonomie se développe alors sur un plan individuel ou groupai, au niveau de la définition de l'activité, de son organisation, de sa mise en œuvre, de sa réalisation. Celle-ci va ainsi pouvoir être objet de négociation et impliquera dans ce cas une régulation conjointe (Reynaud. 1993) ${ }^{20}$ de l'activité collective. En fait, l'ensemble des règles qui structurent la production forme un système cohérent. Ce dernier est le résultat d'une régulation qui provient de l'existence d'espace d'autonomie permettant à chacun de mettre en avant sa propre rationalité, légitimant dans le même temps le dispositif de règles qui structure l'action..J-D Reynaud souligne que : «[...] si Von veut retenir la réalité sociale, il faut parler de régulation plutôt que de règles et la régulation est une caractéristique très générale de l'action sociale, elle est largement constitutive de son caractère rationnel. Elle prend sa source dans le message normatif qu'émet toute action sociale. dans l'offre d'engagement et de réciprocité que comporte toute interaction qui cherche à s'établir » (1993) ${ }^{21}$

L'autonomie ${ }^{22}$ de l'acteur dans le travail est donc plus ou moins "permise» par l'organisation et recherchée par l'acteur (dans la mesure où ce dernier essaye de réaliser un équilibre entre ses propres attentes et celles de l'organisation). 

capacité d'action développée pour s'affranchir de la dépendance aux autres. Pour De Terssac. "À la base de l'accord sur une solution d'organisation il y a une transaction clans laquelle s'échange la reconnaissance mutuelle de l'autonomie et du contrôle» (1992) ${ }^{23}$. C'est pourquoi elle implique un comportement "rationnel» de l'acteur en fonction de la situation sociale dans laquelle il se trouve. Sous cet angle, on peut considérer l'organisation comme un ensemble d'individus dont l'interdépendance n'est jamais totale. Chacun, en arrièrefond, poursuit ses intérêts. Acquérir une autonomie professionnelle (cognitive. organisationnelle. fonctionnelle) signifie augmenter sa marge de manœuvre, sa liberté. Ce qui entraîne forcément une distance entre l'action réellement effectuée et ses modalités d'exécutions définies par l'organisation. L'autonomie ainsi acquise dégage l'acteur de la conduite prescrite pour un processus donné et assouplit les contraintes posées par l'organisation. On conçoit qu'elle puisse dès lors devenir une condition de l'efficacité du travail au sein d'un collectif dans la mesure où elle autorise une régulation entre celui-ci et les acteurs sociaux. Lors de la mise en œuvre de systèmes collaboratifs, la recherche de l'appropriation du dispositif personne-machine-personne semble être un comportement ré-actif à une situation de travail nouvelle. C'est pourquoi la démarche peut se révéler être un processus plus ou moins conflictuel qui rend compte des tensions existantes dans une organisation du travail évolutive.

Lappropriation du dispositif socio-technique est donc également signe d'une stratégie individuelle d'action face au travail prescrit et à son contexte. Ce qui n'est pas pour surprendre. Il convient de se rappeler que les acteurs sont d'abord des construits sociaux, leur démarche d'action est liée à des conditions matérielles, structurelles, humaines du contexte qui définissent et guident leur rationalité (Crozier. Friedberg. i 977) ${ }^{24}$.

Ces éléments forment aux yeux des acteurs autant de cadres en fonction desquelles ils élaborent une stratégie d'action.

\section{Du conflit à l'acteur collectif}

Les situations conflictuelles sont très fréquentes dans le contexte des systèmes collaboratifs professionnels actuels où la communication est souvent formalisée, gérée et contrôlée. C'est pourquoi l'acteur va tendre à modifier l'usage prescrit de la technologie, à contourner les règles prédéfinies et à créer son propre système de régulation. Aussi, des conflits entre les acteurs professionnels et institutionnels, ou professionnels entre eux peuvent-ils émerger à différents niveaux. En effet, dans la mesure où «/.../ une action collective se rapporte à un objectif à réaliser, sa régulation est donc limitée à un domaine déterminé, elle mobilise des individus, et en exclut d'autres" (Reynaud. 1989) ${ }^{25}$ Le champ de l'action sociale est donc un territoire délimité par l'organisation., L'acteur (individuel ou collectifi ${ }^{26}$ ) qui cherche le plus souvent à outrepasser les règles prescrites afin d'étendre son champ d'autonomie entre, à un moment ou un autre, en conflit avec l'organisation ou avec les autres acteurs. Si le conflit est mis à jour, il peut se résoudre par le développement d'un processus de négociation. On peut considérer celui-ci (Fischer. 1990) ${ }^{27}$ «/.../ comme un processus, intervenant généralement à l'occasion d'une relation conflictuelle, au cours duquel s'instaurent des échanges, pour aborder les problèmes qui les opposent et déterminer les modalités de relations acceptables pour les deux termes à l'avenir ». On remarque qu'un ajustement est cherché entre l'action individuelle autonome et l'action individuelle 
prescrite au sein d'une activité sociale, foule activité sociale implique donc une dimension d'action collective.

En résumé, nous avons vu que l'objet d'étude se situe au niveau d'une activité médiatisée par ordinateur dans laquelle la communication est plus ou moins prescrite et formalisée. Pour autant, il s'agit d'une activité spatialement et temporellement distante, articulant des interdépendances individuelles. C'est pourquoi, il semble que la notion de conflit soit particulièrement pertinente comme analyseur de la situation et du processus considérés

La notion d'autonomie est aussi particulièrement intéressante au regard de la problématique. Approfondir la façon dont cette autonomie est proposée aux acteurs (coordination des tâches) et aménagée en retour par ceux-ci (coopération des acteurs dans l'activité) permet de pousser la réflexion. Pour contourner les règles prescrites, ils développent donc un système de communication informelle qui ne correspondra pas nécessairement à la logique de départ définie par l'organisation. Ce phénomène représente pourtant une capacité collective à participer de manière spécifique aux objectifs de l'organisation formelle. On peut affirmer que les normes implicites instaurées individuellement ou collectivement par les acteurs sont l'indice d'une régulation autonome de leur part. Elles ont un double intérêt : destinées à supprimer les sources d'incertitudes (vérifier, comprendre, contrôler), elles sont cependant dans le même temps, à la source d'autres incertitudes. L'incertitude sert ainsi de soubassement à l'action stratégique de l'acteur. En effet, c'est dans cet espace plus ou moins prédéfini que doit se constituer un pouvoir de négociation. Ce pouvoir sert l'intérêt de l'acteur avant même de servir celui de l'organisation (Linhart. 1994) ${ }^{28}$. L'acteur déploiera sa propre logique d'efficacité cl créera, par conséquent, un agencement organisationnel approprié. On peut d'ailleurs supposer a contrario qu'un dispositif de travail qui contraint trop le réseau d'acteurs risque de modifier d'autant plus le processus communicationnel prescrit au profit d'une activité collective imprévue émanant des acteurs eux-mêmes. Dès lors, c'est la convergence entre la finalité de l'action et la constitution du groupe qui permet d'instituer un acteur réellement collectif autour du processus de régulation: cette convergence doit émaner de la volonté de l'organisation et de celle de ses membres. Elle repose sur un ensemble de règles, formelles et informelles, en lien avec la constitution d'un groupe social, d'un acteur collectif. Elles constituent l'identité du groupe, au-delà de sa diversité (Reynaud. 1989) ${ }^{29}$. C'est donc à partir de ce groupe qu'émerge et s'élabore progressivement une construction sociale qui gère l'ensemble des stratégies individuelles et régule les interactions des acteurs.

\section{À la recherche de l'interaction collaborative}

Finalement, les analyses des terrains nous ont amené à mettre en évidence des caractéristiques de la communication médiatisée et de la construction du lien interpersonnel communes :

Notamment, comme souligné par les analyses sociopragmatiques des terrains, on doit insister sur I importance de reconfigurer les modalités d'établissement des liens interpersonnels distants de manière spécifique au nouvel environnement de travail : la médiatisation des activités a montré que les processus communicationnels en jeu lors d'une activité professionnelle en présentiel de cette activité. Fn fait, au-delà des processus de relations interpersonnelles au cours du travail, la réalisation réussie de 
l'activité repose sur la mise en œuvre et la construction d'un acteur effectivement collectif : c'est-à-dire qu'il faut que pour lui ne se pose plus la question des barrières techniques, spatiales, temporelles et culturelles d'une activité, mais seulement la réalisation conjointe d'une activité professionnelle désormais distante. Il y a là tout un processus de mise en œuvre du projet à penser d'un point de vue organisationnel et managérial.

Par ailleurs, les analyses ont montré l'importance, au-delà d'une approche volontariste des acteurs, d'une organisation du travail cohérente : nous avons vu qu'il pouvait être intéressant de travailler, pour certaines activités, collectivement. Cependant, l'idée de travail collectif, à défaut d'être réellement collaboratif, implique au minimum une structuration des activités autant que des processus communicationnels - probants pour mutualiser les connaissances et développer de nouvelles compétences. Les processus communicationnels qui lient les personnes au cours d'une activité professionnelle sont essentiels dans une activité distante médiatisée dans la mesure où nous l'avons particulièrement noté avec les analyses sociopragmatiques ces processus permettent de recréer une situation la plus proche possible d'une situation de face-à-face généralement connue des professionnels et considérée comme une forme appropriée d'organisation du travail collectif. Les structures communicationnelles sont les vecteurs des marques sociales véhiculées dans toutes activités collectives. Elles sont donc primordiales pour la mutualisation des connaissances. Ces structures communicationnelles supposent de lier les personnes de telle sorte qu'un réel maillage est créé au travers duquel se construit peu à peu un acteur collectif. À partir de là, les systèmes collaboratifs peuvent être envisagés comme favorisant la mise en synergie des acteurs d'un groupe de travail.

Cela dit. la construction d'un acteur réellement collectif demande l'implication des acteurs individuels, au niveau de la motivation: la question de l'intérêt individuel et collectif de la médiatisation d'une activité professionnelle est primordiale: on sait que tout changement technico-organisationnelle suppose un investissement des acteurs pour l'appropriation du dispositif, l'engagement dans de nouvelles procédures de travail : ce qui implique la motivation et la participation de chacun sans lesquelles la reconfiguration des relations professionnelles dans une autre organisation du travail ne peut aboutir pleinement.

43 Ce postulat est également présent au niveau des compétences nécessaires pour " taire fonctionner » un réseau de communication, qu'il s'agisse de compétences concernant le dispositif technique, ou de compétences concernant la médiation des connaissances. Il s'agit donc de porter également la réflexion sur l'importance de l'acquisition des nouvelles compétences pour la réussite distant médiatisé.

Dans le travail de groupe, nous savons qu'une activité peut être individuelle ou supposer le travail de plusieurs personnes IIy a la une certaine granularité,une progression par valeurs successives: l'activité peut être fragmentée en différentes formes organisationnelles celles-ci s'ajoutent les unes aux autres (coordination, puis coopération impliquant la coordination) générant différents degrés de travail collectif Cette granularité est elle-même induite par le dispositif socio-technique utilisé pour la réalisation du travail, fa possibilité de réaliser :

45 - une activité plurielle (nous prenons « plurielle » dans l'acception la plus usuelle qui est celle d'une activité impliquant un nombre de personnes supérieur a une imite). 
- ou une activité collective (nous utilisons le terme «collectif» pour évoquer des personnes constituées en groupe).

- ou une activité collaborative (impliquant un travail conjoint), dépendant bien entendu, pour partie du système collaboratif dans la mesure où les dispositifs techniques asynchrones et les dispositifs synchrones ne favorisent pas la création de liens de même nature entre les acteurs les interactions entre ceux-ci sont plus ou moins denses et ne revêtent pas la même qualité De ces relations dépend la réalisation plus ou moins conjointe de l'activité, c'est-à-dire de la construction d'un acteur collectif Cette conclusion est évidemment a pondérer en fonction de tous les cléments inhérents aux situations de travail médiatise et constituant le contexte de communication

On comprend bien en quoi les systèmes de travail collaboratif peuvent être des outils d'aide à la structuration des activités professionnelles d'un collectif de travail. On sait que les groupwares impliquent un système d'échanges régulés qui génère des dépendances informationnelles et cognitives mutuelles. Celles-ci doivent, logiquement, stimuler une coopération entre les individus, donc améliorer le rendement, et par feed-back réduire les incertitudes des organisations. Cette structuration des contextes d'actions mêle comportements individuels et collectifs. On passe donc à un niveau intermédiaire entre l'organisation prescrite des activités et la réalisation de celles-ci par les acteurs. Cette dimension organisationnelle constitue un des contextes d'action primordiaux des acteurs professionnels.

\section{Conclusion}

Ainsi, on peut prendre en compte une série d'effets et de relations qui ont pour objet de penser l'organisation d'une activité comme un processus dynamique, produit de rapports interpersonnels. C'est-à-dire que nous considérons ici l'organisation d'une activité distante médiatisée non seulement comme éventuelle productrice de services (au sens économique du terme) mais également de cultures, de projets, de tensions, de régulations. Cette dynamique des relations interpersonnelles, sous tendue par un management adapté, peut développer un jeu collectif par le biais de l'existence d'interactions qui favorisent la synergie de compétences sociales et professionnelles, par la création de liens d'interdépendance entre acteurs autonomes, et des compétences, (sur le plan organisationnel ou cognitif). Ces interdépendances supposent, cependant, que chacun participe réellement à l'action collective et qu'il y ait de ce fait, des échanges négociés au cours desquels un acteur collectif se met en place pour réaliser une activité collaborative au plein sens du terme, fondement d'un construit socio-cognitif de qualité. 


\section{BIBLIOGRAPHIE}

COMTET. I., Systèmes collaboratifs et acteurs professionnels en réseau Je communication. Thèse de doctorat de Sciences de l'Information cl de la Communication sous la direction du Pr Josiane JOUET, Université Panthéon-Assas. Paris II. IW)

CROZIER. M. FRIEDBERG. E. L'acteur et le système. Paris : Le Seuil. 1977.

DE TERSAC. G. Autonomie dans le travail. Paris : PUF. 1992.

FINHOLT. I. SPROULL., L KlESLER S. Communication and performance in Ad Hoc task groups. Intellectual Team Work. New Jersey : LEA. 1995.

FISHER. G. Le champ du social. Paris : Dunod. 1990.

FRIEDBERG E. Le pouvoir et la règle, les dynamiques de l'action organisée. Paris : Le Seuil. 1993.

GALEGHER, C. KRAUT, R. EGIDO. C, Pattems of contact and communication in scientific research collaboration. Intellectual Team Work. New Jersev : Il A. 1995.

GOFFMAN. E. Mise en scène de la vie quotidienne. Présentations de soi. Paris : Les Editions de Minuit. 1973

LAFAYE. C. La sociologie des organisations. Paris : Nathan. 1996

LINHART.D (1994). La modernisation des entreprises. Paris : la Découverte, Coll. Repères. 1994 OPPER, S FERSKO-WEISS. H. Technology for teams. New York : Van Nostrand Reinhold. 1992 PERIN. P. GENSOLLEN. M. La communication plurielle, l'interaction des téléconférences, Paris. La Documentation française. 1992.

REYNAUI)., J-D. Les règles du jeu l'action collective et la régulation sociale. Paris : Armand Colin. 1993

\section{NOTES}

1. Galegher C. Kraut K. Egido C, (1995). Patterns of contact and communication in scientifie research collaboration. Intellectual TeamWork. LIA. New Jersey

2. Nous mettons ici sur le même plan les notions «d équipe » et de "groupe constitué »

3. Crozier M. Friedberg E. (1977). L'acteur ci le système Les contraintes île l'action organisée, Le Seuil. Paris

4. Traduction I.Comtet « Knowing the capabilities of the people you're working with on the team helps Groupware facilitates this by improving communications If the flow of information / / is fluid and efficient, then individuals can contribute the best of themselves "

5. Opper S. Fersko-Weiss H. (1992) Technology for teams. Van Nostrand Reinhold. New York, p. 29

6. Goffman E. (1973). Mise en scène de la vie quotidienne. Présentations de soi. I es Editions de Minuit. Paris

7. Périn P, Gensollen M. (1992). La communication plurielle: l'interaction des téléconférences, La Documentation française, Paris

8. Synchrone ou en temps réel 
9. Quasi-synchrone ou supposant un court laps de temps de réponse comme la « chat-box » par exemple

10. Asynchrone ou en temps différé

11. Finholt T Sproulll L Kiesler S. (1995). Communication and performance in Ad Hoc task groups, Intellectual Team Work. LEA New Jersey

12. Traduction $1:$ " [.] there probably was a relationship between managerial ability and volume of group computer mail : [: that is good managers determined haw to exploit the technology to benefit the group directly".

13. Crozier M. friedberg E, I 1977), l'acteur et le système. le Seuil. Paris. pp.88-89

14. De Terssac G (1992)., Autonomie dans le travail PUF. Paris.

15. Lafaye C. (1996).La sociologie des organisations. Nathan. Paris, p 77

16. Crozier M. Friedberg E (1977). L'acteur et le système. Le Seuil. Paris, p 197

17. Friedberg E. (1993). Le pouvoir et la règle les dynamiques de l'action organisée.

18. Fisher GN. (1990). Le champ du social. Dunod. Paris, p. 234.

19. friedberg L. (1993). Le pouvoir et ta règle, les dynamiques de l'action organisée. Le Seuil. Paris, p. 229

20. Reynaud.JD. (1993). Les règles du jeu, l'action collective et la régulation sociale. Armand Colin. Paris

21. Reynaud.JD (1993). Les règles du jeu l'action collective et la régulation sociale Armand Colin. Pans, préface $\mathrm{p} . \mathrm{V}$

22. C'est-a-dire la possibilité de décider par soi-même

23. De Terssac G (1992) Autonomie dans le travail PUF Paris p. 183

24. Crozier M. Friedberg E. (1977). L'acteur et le système. Le Seuil. Paris.

25. Reynaud.11). (1993), Les règles du jeu l'action collective et la régulation sociale. Armand Colin. Paris. p. 99.

26. Nous prenons le terme « acteur collectif» dans le sens de.JD Reynaud. (1993). Ibid : l'acteur collectif est défini par une finalité, une intention, une orientation d'actes, régulée par un ensemble de règles, produit des systèmes de règles de chaque acteur

27. Fisher GN. (1990). Le champ du social. Dunod. Paris. p. 152.

28. Linhart I). (1994), La modernisation des entreprises. Coll. Repères, La découverte Paris

29. Reynaud.JD. (1993). Les règles du jeu l'action collective et la régulation sociale. Armand Colin. Paris

\section{AUTEUR}

\section{ISABELLE COMTET}

École de Management, Grenoble 